\title{
Digital Library Research (1990-2010): A Knowledge Map of Core Topics and Subtopics
}

\author{
Son Hoang Nguyen \\ Information \& Knowledge Management, University of Technology Sydney, NSW, Australia \\ \{Hoang.S.Nguyen@ student.uts.edu.au\}
}

\begin{abstract}
Research and development in digital libraries (DLs) have proliferated over the past two decades, producing many research publications on different topics. However, there is no comprehensive list of core research topics and subtopics that can be used as a knowledge map of digital library (DL) research. Based on an analysis of the call for papers from 37 conferences volumes of three major international DL conferences (JCDL, ECDL, ICADL), a list of 15 core topics and 210 subtopics was created. Searches were conducted on the SCOPUS database using the list of topics to validate and expand the list. The resulting list of topics was structured into a DL knowledge map comprising a list of 21 core topics and 1015 subtopics representing research in DLs. This study will provide a panoramic view of DL research for DL researchers, practitioners and educators. It can be used to create a visual knowledge map of DL research, and can also be used to develop a new DL curriculum.
\end{abstract}

Keywords: Digital Library Research, Knowledge Map, Research Topics, Core Topics, Subtopics, Curriculum Development, Digital Library Ontology

\section{Introduction}

Knowledge maps, commonly referred to as mind maps, semantic networks, and concept maps, play an important role in the construction, learning, and dissemination of knowledge (http://technologysource.org/extra/83/ definition/2/) . It is a tangible representation or catalog of the concepts and relationships of knowledge that enables a user to find the desired concept, and then retrieve relevant knowledge sources (home.earthlink.net/ ddstuhlman/ defin 1.htm). In term of application, the knowledge maps may involve developing an ontology (semantic relationships of concepts), conducting social network analysis, executing a survey, engaging a group of people in sensemaking, action research or ethnography, etc (http://kmwiki.wikispaces.com/ Knowledge+mapping). In particular, in the field of information science and computer science (semantic web development), an ontology formally represents knowledge as a set of concepts within a domain, and the relationships between those concepts. It can be used to reason about the entities within that domain, and may be used to describe the domain http://en.wikipedia.org/wiki/Ontology_\%28computer_science\%29

Regarding the DL domain, since its inception as a new field of study about two decades ago, research and development activities in DLs have grown quite significantly, drawing researchers and practitioners from a range of fields including Computer Science (CS) (63\%) and Information Science \& Library Science (LIS) (26\%) (Chart1). A search on SCOPUS database reveals a dramatic rise in the number of publications (articles, papers, review, etc) from 436 publications in1990-1999 to 7469 publications in 2000-2010 (Chart 2).

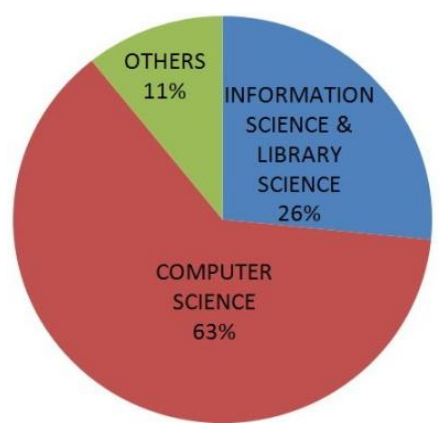

Chart 1: Distribution of DL fields (1990-2010) retrieved from Web of Science ISI on (14/05/2011)

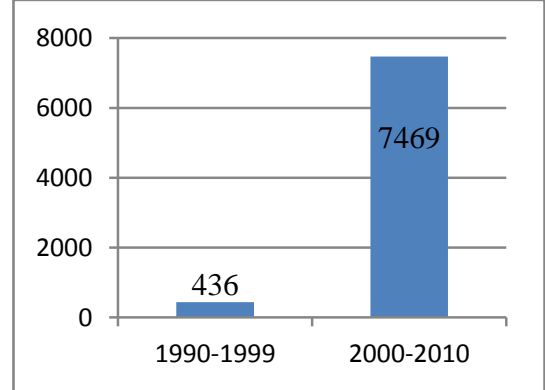

Chart 2: Rise in DL publications (1990-2010) retrieved from SCOPUS on (14/05/2011) 
Although research in DLs has been spearheaded by computer and information scientists, researchers from many other fields have also joined hands to look into several research issues that form part of, or surround, DLs. Consequently the field of DLs has been defined differently by different groups of people, and often the connotations vary quite significantly (see [2],[3],[5-7], [13]). While the different viewpoints of DLs have brought diversity and innovations, to date there isn't any knowledge map of DL topics providing a comprehensive view of the field. This paper discusses the methodology and findings adopted in a research designed to develop a knowledge map of DL research.

\section{Literature Review}

Various research topics in DLs have been identified by researchers in the late 1990s and early 2000s (see for example [2],[3],[5-7], [13]) that have been widely used in research and education in DLs around the globe. The DL research topics, as defined by Hsinchun Chen in 2005, represent a view of information technology in which social impact matters as much as technological advancement [5]. The field of DLs is the meeting point of many disciplines and fields, including computer science, data management, information retrieval, library sciences, document management, information systems, web, image processing, artificial intelligence, human-computer interaction, and digital curation [4]. While reviewing research and development in DLs in the nineties, Chowdhury and Chowdhury (1999) grouped DL research into 16 areas [7]. More recently, two research groups have attempted to find out the core topics in DLs: the first research was conducted by Pomerantz et al (2006) on a sample of 1064 DL publications (1995-2006) that produced 19 modules (core topics) and around related 69 topics [14]; and the second was conducted by Liew (2008) with 557 publications (1997-2007), producing 5 themes (core topics) and 62 related or subtopics [12]. They both provided fundamental frameworks of DL core and subtopics, with Pomerantz et al (2006) covering core CS and LIS topics, and Liew (2008) providing an insightful view of organizational and people issues of DL research. The basic tenet of the research reported in this paper is that there is the need for a more comprehensive research with a larger sample covering the field of DL research from the beginning. The major objective of the ongoing research, part of which is reported here, is to identify the core topics and subtopics of DL research (1990-2010) for generating a DL knowledge map.

\section{Methodology}

As stated earlier, the main objective of this research was to build a knowledge map of DL research topics. Therefore, the research issues to be considered in this study involved identification of the core topics and subtopics in DL research which then could be used on the one hand to develop a DL knowledge map and also to study the evolution of research in the field. The first challenge facing this study was the lack of a knowledge organization system for DLs. Therefore a new methodology had to be designed to build a concept map of DLs. Literary warrant was taken to be the guiding principle and a multi-stage development approach was taken (Figure1).

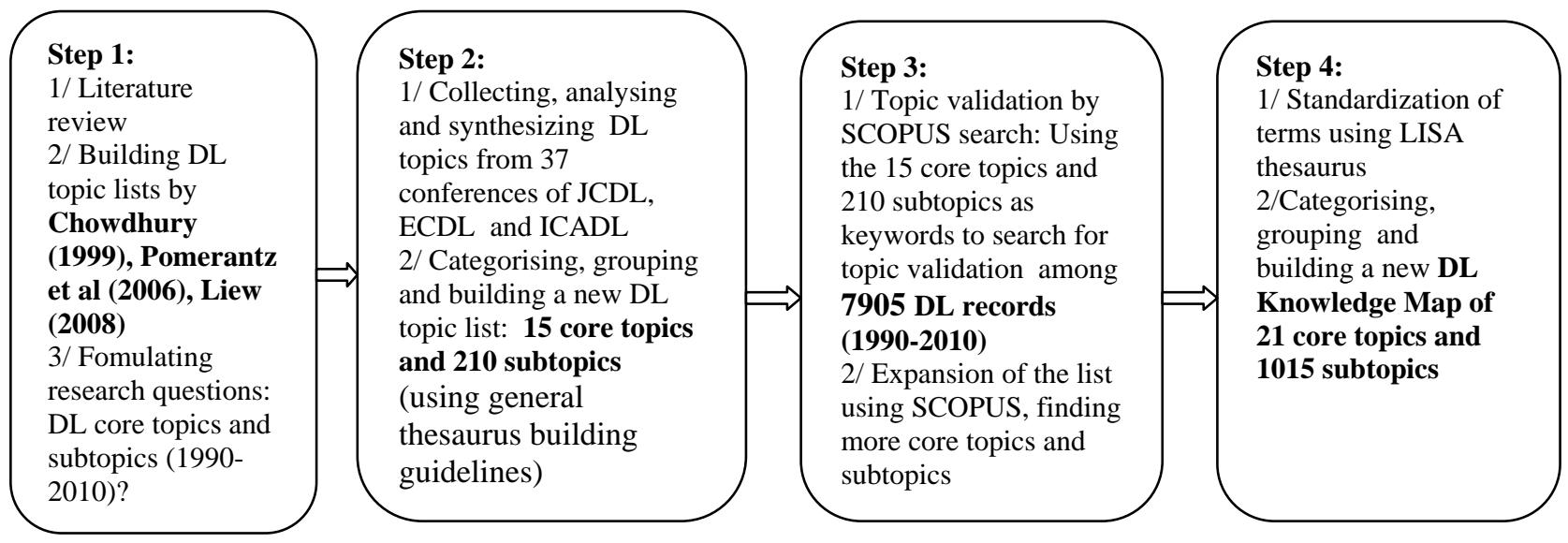

Figure1: 4 Steps of Research Process

Step 1: A 1 list DL research topics and subtopics was created, based on the literature review, especially from the findings of Chowdhury and Chowdhury (1999)[7], Pomerantz et al (2006)[14] and Liew (2008)[12]. However, these studies provided lists of core topics and subtopics according to the viewpoints of individual researchers, and they were limited by the selection of literature studied by the concerned researchers and their study objectives, etc. 
As a result, it was realized that any list of core topics and subtopics prepared on the basis of these three studies would not truly represent the field of research. Furthermore the list of topics and subtopics from these studies shows more differences than commonalities, and it paved the way for further research and investigations (Steps 2 and 3).

Step 2: Keeping in view the principle of literary warrant, call for papers (CFPs) for three international conference, viz. JCDL, ECDL and ICADL [8-10] were chosen for this study because these international conferences are the platforms where researchers report on their new research findings. The editorial team (Programme Committee) comprises recognized experts in the field who bring out the CFPs. In this research, the CFPs covering various DL topics from 37 conference volumes: JCDL (2001-2010), ECDL (1997-2010) and ICADL (1998-2010) were collected from the conference websites.

List of core topics and subtopics in each conference call was noted, and by combining these DL topic lists with those of earlier studies, a table of 15 core topics and 210 subtopics were created (Table 1)The list of core topics and subtopics was structured by using the general guidelines for thesaurus building [1]. However, as opposed to a typical knowledge organization scheme like a classification scheme or a thesaurus, the DL knowledge map comprised a list of core and subtopics where each core topic has a list of subtopics, and some subtopics appear under more than one core topics. The reason for taking this approach was that the DL knowledge organization system was primarily designed to be a tool for showing the concept map and research in the field, and in such a tool a given topic, for example information retrieval, may appear under different topics like user studies, collection management, digital library architecture, etc. depending on the context of research. This is discussed further in Step 3.

In preparing the table of 15 core and 210 subtopics, the following steps were followed:

- Building a draft table of core topics, then gathering their subtopics from the CFPs which were subsequently checked and verified with the resulting conference volumes

- The core topics are defined as the broader semantic scopes of terms (Broader Terms) while their subtopics are defined as narrower semantic scopes of terms (Narrower Terms)

- The core topics and their subtopics are linked by semantic relationships. Some subtopics can appear under more than one core topic because of their semantic cross-relationships, e.g. the subtopic Interoperability is related to core topics Information Retrieval and Architecture - Infrastructure.

- The original terms and phrases of all of the core topics and subtopics from the CFPs were kept because the language and terminologies used in the CFPs were sometimes loose and varied from one conference call to another, e.g.: Archives, Archiving;, Preserving, Preservation; Filter, Filtering; Ebooks, Electronic Books, etc. These were standardized and/or extended in Step 3 (see below)

- Knowledge and understanding of the DL field was used for indentifying and classifying the topics.

- A period of 1 month (April 2010) was spent for gathering and analysing the data from the 37 CFPs.

\section{Problems/Issues}

Although the CFPs from 37 conferences provided a good picture of the field of DL research activities around the globe, it was considered that limiting this study only to this approach would suffer from two major drawbacks:

1. because of the limited capacity of a conference volume in terms of accommodating published papers, DL conferences can only provide a snapshot of research in the field, and therefore they cannot provide a representation of the entire field of research, and

2. often researchers are constrained by the fact that they need to submit papers within the framework of the CFPs and therefore (a) many cannot report their research in conferences because of the incompatibility of their research and the CFPs, and (b) the length and breadth of the DL research field, which is multidisciplinary in nature, cannot be properly reflected only through an analysis of the conference papers.

It was therefore decided that the principle of literary warrant could be observed properly if a large representative database was used to verify and expand the list of 15 core and 210 subtopics, generated through the first phase of the study, and this would help us generate a larger and more comprehensive knowledge map of DLs.

Step 3: SCOPUS database was chosen because of its being the largest abstract and citation database of peerreviewed literature [15]. A search for DL publications on the SCOPUS database was conducted during March 2011 that produced 7905 publications (1990-2010). The list of 15 core and 210 subtopics was used as a set of keywords to conduct a series of searches within 7905 publications in order to validate DL topics and identify more keywords that could be used as core topics or subtopics. The process is explained below: 
- For example: The topic "Digital collections" was used as a keyword for searching which produced 53 hits. In each record, there were always 2 clusters of keywords - Author keywords \& Index Keywords, such as: Author keywords (Digital libraries; Information dissemination; Information services; Library collections development), Index Keywords (Core journals; Digital collections; E-books; Institutional repositories; Library collections development; Multimedia database; Relationship management; Strategic plan; University libraries). The topic "Digital Collections" was defined as a valid and standard term for it found 53 records. Topics that generated no results, such as: "Digital Library Creation" or "Disseminating Asian unique and indigenous knowledge and culture", etc, were excluded for being invalid terms.

- Because of time limitations, all of the new keywords that were found within the first 5 records were included in the list. By collecting new keywords that appeared in Author keywords \& Index Keywords from each record, more DL core topics and subtopics were found.

- By using this method, the DL topic list were enlarged with 21 core topics and 1015 subtopics.

Step 4: The 21 core topics and 1015 subtopics were checked with LISA for topic standardization

- Although the research objective was to create a broad DL knowledge map, not thesaurus building, some techniques of the thesaurus building [1] were used to organize the terms.

- There were some principal thesaurus building methods used:

1. Defining the scope, or meaning, of terms: this is a very important process for defining the core topics (as Broader Term to which another term or multiple terms are subordinate in a hierarchy) and subtopics (as Narrower Terms that is subordinate to another term or to multiple terms in a hierarchy ) [1], e.g.: The topic cluster below is hierarchically structured by a Broader Term "Storage" and its 9 Narrower Terms

\begin{tabular}{|l|l|}
\hline Broader Term & Storage \\
\hline Narrower Terms & $\begin{array}{l}\text { Digital Storage, Storage systems, Storage devices, Storage media, Storage technology, } \\
\text { Storage management, Hierarchical storage, Data storage equipment, Digital Image Storage }\end{array}$ \\
\hline
\end{tabular}

2. Furthermore, semantic relationships were used to categorize and group terms [1], e.g.:

\begin{tabular}{|l|l|}
\hline Relationship Types & Examples \\
\hline Equivalence & Electronic books / ebooks \\
\hline Synonymy & Filter / Filtering; Archive / Archiving/ Archives \\
\hline Lexical variants & Information Retrieval / Search \\
\hline Near synonymy & Multimedia / Music, Video, Document \\
\hline Hierarchy & Storage / Storage systems, Storage devices, Storage management \\
\hline Generic & Social Sciences/ Art, Culture \\
\hline Instance & \multicolumn{2}{|l|}{} \\
\hline Whole / Part & Disaster/ Digital Preservation \\
\hline Associative & Knowledge Management/ Knowledge discovery, Knowledge acquisition, \\
\hline Cause / Effect & Knowledge Management/ Knowledge Economy \\
\hline Process / Agent & Research / Scientist, Scholar \\
\hline Action / Product & Web 2.0/ Library 2.0, Information Literacy 2.0 \\
\hline Action / Target
\end{tabular}

\section{Findings:}

Table 1 shows the 15 core and 210 subtopics, identified from the 37 conference volumes: JCDL (2001-2010), ECDL (1997-2010) and ICADL (1998-2010). The table shows the conference name and the year the topic first appeared in the CFPs e.g., 1.Digital Library Creation, 2. Digital Video Libraries, 3. OCR Text Verification (ICADL,98): the three topics first appeared in ICADL in 1998. All of the terms were kept originally in form of structured - table of DL core and subtopics for later research. Each group of subtopics of the same conference and year is separated by Slash sign (/) 


\section{Table 1: 15 CORE TOPICS AND 210 SUBTOPICS ON DIGITAL LIBRARY RESEARCH (1997 - 2010)}

1. Digital Collections (27 subtopics): 1.Digital Library Creation, 2. Digital Video Libraries, 3. OCR Text Verification (ICADL,98)/ 4. Arts Collection, 5. Government Information Collection, 6. Manuscripts Collection, 7. Humanities Collection, 8. Geographic Material Collection 9. Authoring, 10. Watermark, 11. Image Processing (ICADL,99)/ 12.Multimedia, 13. Agents (ICADL,99; ECDL,98;JCDL,01)/ 14.Electronic publishing, 15. Electronic journals , 16. Electronic newspapers (ICADL,00; ECDL,00; JCDL,01) / 17. EBooks (ECDL,02) / 18. Management (Collection), 19. Integration (Collection) (ECDL,03) / 20. Music Digital Libraries (JCDL,01; ECDL,04) / 21. Heterogeneous digital collections (ICADL,04)/ 22. Scholarly publishing (ICADL,05) / 23. Concepts of Digital Libraries, 24. Concepts of Digital Documents (ECDL,05) /25. Large-Scale Collections (JCDL,07)/ 26.Evaluation of Internet Resources (ICADL,98)/ 27. New Publishing (ECDL,00)/

2. Digital Preservation (16 subtopics): 1.Archives (Archiving) (ICADL,99; ECDL, 2000; JCDL,2001) / 2. Cultural Heritage Preservation (ICADL,01) / 3. Web Archiving, 4. Online Archive (ECDL,02) / 5. Digital Archives, 6. Digital Museums (ICADL,02) / 7. Preserving (ICADL,04) / 10. Digital Curation (ECDL,07; JCDL,07)/ 11. Methodological (Digital Archiving - Preservation), 12. Technical (Digital Archiving - Preservation), 13. Legal Issues (Digital Archiving - Preservation) (ECDL,07) / 14. Preservation Systems, 15. Algorithms (ICADL,2010)/ 16. Open Archives Initiative (OAI) (ECDL,04; ICADL,04)

3. Information Organization (42 subtopics): 1.Metadata, 2. Structured Documents (ECDL,97; ICADL,98; JCDL,01) / 3. Categorization (ECDL,99; ICADL,08) / 5. Text Processing, 6. Data Management (ICADL,98)/ 7. Automatic Indexing (ICADL,99; ECDL,00)/ 8. Data Mining (ICADL,98;ECDL,01) / 9. Capturing; 10. Indexing, 11. Cataloging (ECDL,00)/ 12. Harvesting, 13. Interoperability, 14. Scalability, 15. Extensibility Issues, 16. Files/ Formats Representation (ICADL,01)/ 17. Interpretation, 18. Annotation of Documents (ECDL,01)/ 19. Web Cataloguing (ICADL,02)/20. Personalized Classification (ECDL,02) / 21. Information Organization (ECDL,05) / 22. Semi Structured Data (ICADL,05; ECDL,09) / 23. Web-page Analysis (ICADL,05)/ 24. Taxonomy (ICADL,07)/ 25. Named Entities, 26. Links (JCDL,06)/ 27. Classification (ICADL,99; ECDL,00; JCDL,06)/ 28. Scalability; 29. Networked Resource Discovery, 30. Collaborative, 31. Distributes Authoring, 32. Resources Management (ECDL,00)/ 33. Naming Scheme (ICADL,99)/ 34. Organizing Asian Unique and Indigenous Knowledge and Culture (ICADL,04)/ 35. Information Extraction (ICADL,99; JCDL,07)/ 36. Thesaurus, 37. Summarization / 38. Personalisation, 39. Annotation (ECDL,04) / 40. Information Mining in Multilinguality (ECDL,2010)/ 41. Navigation, 42. Querying (ECDL,98; ICADL,00)/

4. Information Retrieval (16 subtopics): 1. Information Retrieval (ICADL,98; ECDL,97; JCDL,01) / 2. Multilingual Information Retrieval (ECDL,97; ICADL,99; ECDL,00) / 3. Cross language, 4. Modelling, 5. Accessibility, 6. Connectness (ECDL,99)/ 7.Cross-Language Filtering, 8. Dissemination; 9. Multicultural (ICADL,99; ECDL,00) / 10. Information Retrieval in Asian Languages (ICADL,03) / 11.Multimedia Information Handling (ECDL,06) / 12. Searching (ICADL,01)/ 13. Access (ECDL,99; ICADL,08) / 14. Disseminating Asian Unique and Indigenous Knowledge and Culture (ICADL,04)/ 15. Distributed Access (ICADL,99; JCDL,07) / 16. Interoperability (ICADL,00)

5.Human - Computer Interaction (12 subtopics): 1.Supporting User Interaction (ECDL,97)/ 2.User Adaptation (ECDL,98)/ 3.Use of Digital Video Libraries (ICADL,98)/ 4.Learning Model, 5.Instruction Model, (ICADL,99) / 6.User Interfaces (ICADL,01;ECDL,99; JCDL,01)/ 7.Evaluation, 8.Usability (ICADL,01; JCDL,01;ECDL,02)/ 9.Portals (ICADL,04)/ 10.Localization (ICADL,05)/ 11. Log Data (ECDL,09)/ 12.Visualisation (ICADL,99; JCDL,06; ECDL,2010)/ 13. Search behaviour (ICADL,00)

6.User Studies (4 subtopics): 1. User Feedback Evaluation (ICADL,99) / 2.Information Needs (ICADL,00) / 3.User's Models (ICADL,01;ECDL,99; JCDL,01)/ 4.User Communities (ICADL,02; JCDL,01; ECDL,01)/

7.Architecture - Infrastructure (13 subtopics): 1. Architectures (ECDL,97; ICADL,99; JCDL,01)/2. Cluster Computer (ICADL,98)/ 3. Network (ICADL,99)/ 4. Interoperability (ECDL,00)/ 5. Agents and tools, 6. Database design \& 7. Database development (ICADL,01)/ / 8. Grids (ICADL,04; ECDL,09)/, 9. Open Source tools and systems (ICADL,08)/ 10. Time and Space (JCDL,06)/ 11. Distributing Digital Libraries (ICADL,98)/ 12. Intelligent Agent (ICADL,99; JCDL,07) / 13. Data Warehousing (ICADL,98)

8. Knowledge Management (8 subtopics): 1. Knowledge Management (ICADL,00; ECDL,01)/ 2. Ontologies, 3. Knowledge Organization Systems (JCDL,07; ECDL,09)/ 4. Knowledge sharing (ECDL,99)/ 5. Content Management, 6. Access Control (ICADL,99)/ 7. Knowledge Representation (ICADL,99;ECDL,99;JCDL,01)/ 8.Knowledge Discovery (ICADL,99; JCDL,07)

9. Digital Library Services (5 subtopics): 1.Interoperability across different services (ECDL,02)/ 2.Ontology-based Services (ICADL,02)/ 3.Personal Digital Libraries (ICADL,04; ECDL,04; JCDL,07)/ 4.Live Reference Service (ICADL,04)/ 5.Mobility Services (ICADL,04; ECDL,2010; JCDL,08)/

10. Web Development \& Applications (Social Web, Semantic Web) (6 subtopics): 1.Semantic Web (ICADL,02; ECDL,09)/ 2.Ontologies, 3.Topic Maps (ICADL,04) / 4.Social Networking, 5. Web 2.0 Technologies (JCDL,07; ICADL,07; ECDL,08)/ 6.Social Media (ICADL,08)

11. Intellectual Property - Security - Privacy (7 subtopics): 1.Intellectual Property, 2.Rights Management (ECDL,00; ICADL,01; JCDL,01)/ 3.Authentication, 4. Authorization (ICADL,99)/ 5. Cryptography, 6. Compression (ICADL, 03)/ 7. Security (ICADL,99)

12. Cultural, Social, Legal , Economic Aspects (16 subtopics): 1.Social Aspects, 2.Management, 3.Policy Issues (ICADL,08; JCDL,01)/ 4. Cultural Issues, 5. Social Issues, 6. Legal Issues, 7. Economic Issues, 8.Education (ICADL,99; ECDL,06) / 9. Economic Models, 10.Terms and Conditions for Access and 11.Use (ECDL,00)/ 12.Scholarly Communication, 13.Pricing (ICADL,01)/ 14. Pedagogical Issues (ICADL,02)/ 15.Exploring a Cross-Culture Context for DL studies in Asia, 16. Social impacts of DL in Asia Technology (ICADL,04)/

13. Digital Library Applications (23 subtopics): 1.Digital Library Applications (ICADL,98)/ 2.Digital Libraries and Education (ICADL,99;JCDL,06; ECDL,03)/ 3.E-learning, 4.Mobile Learning (ICADL,03)/ 5.e-science (ICADL,05)/ 6.e-government (ECDL,03; ICADL,07)/ 7.Applications Libraries, 8.Museums, 9.Archives, 10. Health Care and Medicine, 11.Digital Earth/Geospatial, 12.Law, 13.Art, 14.Music,15.Humanities, 16.Culture, 17.Social Sciences, 18.News, 19.Current Affairs, 20.Information Industry, 21.Environmental Monitoring, 22.Natural Sciences, 23. E-commerce (ECDL,03)

14. International Collaboration \& Development (14 subtopics): 1.Development of Universal Digital Libraries (ICADL,98)/ 2.International Collaboration (ICADL,00; ECDL,00)/ Collaboration of 3.Computer Science, 4.Information Science, 5.Librarianship, 6.Archival Science and Practice,7. Museum Studies and Practice, 8. Technology, 9. Medicine, 10.Social Sciences, 11. Humanities, 12.Academe, 13.Government, 14.Industry (JCDL,01)/ 
Table 2 shows a DL knowledge map of 21 core topics and 1015 subtopics found from 7905 DL publications (19902010) in SCOPUS. For each core topic, there are several clusters of subtopics and the number of publications is assigned for each subtopic, e.g., Collections(363): there are 363 publications on the topic .

Table 2: A Digital Library Knowledge Map of 21 core topics and 1015 subtopics (1990-2010)

\section{Core Topic 1: Digital Collections (48 subtopics):}

Cluster1. Collections(363): Resources(603), Digital documents(41), Digital information(57),Data Collection(28), Information Sources(26) Cluster2. Acquisition(432): Collection development(35), Collection development policy(3), Content creation(8), Digitization(58), Digitisation workflow(1), Resource Sharing(15)

Cluster3. Database(General)(1210): Web Databases(13), Video Database(14), Image Database(29), Music Database(8)

Cluster4. Collection management(50): Collection Evaluation(2), Information Evaluation(2), Resources Management(46)

Cluster5. Multimedia(General)(496): Multimedia collections(6), Multimedia contents(6), Document collection(33), Text collection(5),

Electronic books/ebooks(51), Electronic Publishing(251), Electronic Journals(85),Electronic Newspapers(1), Newspapers(18), Music(112),

Digital Music Libraries(26), Video(246), Digital Video Library(16), Photos(24), Manuscripts(32), Scholarly Publishing(12), Scientific resources(1), Scientific Data(12),Humanities(Collection)(20), Government Information(6), Government documents(3), Heritage collections(4), Arts Collection(1), Digital talking books(3), Audio(73), Educational Resource(29), Video Game(6)

Core Topic 2: Digital Preservation(46 subtopics):

Cluster 1. Preservation(General)(174): Preservation management(2), Preservation Policy(2), Preservation technologies(1), Preservation Process(1), Long-term preservation(19), Cultural Heritage (Preservation)(60), Historic Preservation(16), Digital Museums(13), Restoration(14), Recovery(20), Migration(24), Algorithms(Preservation)(4), File formats(20), Disaster(12), Recovery(20), Disaster recovery(4), Data recovery(2), Data protection(2), Life-cycle management(4), Error Recovery(2), Curation(22)

Cluster 2. Storage(General)(634): Digital Storage(160), Storage systems(13), Storage devices(2), Storage media(4), Storage technology(2), Storage management(5), Hierarchical storage(1), Data storage equipment(152), Digital Image Storage(136),Distributed storage resources(3) Distributed Storage(6)

Cluster 3. Archives(General)(281): Archives management(30), Web Archiving(6), Online Archive(5), Open Archives Initiative(50), Data Archive(4)

Cluster 4. Repositories(General)(211): Institutional Repositories(32), Online repositories(3), Open source repositories(2), Remote repositories(1),Learning Object Repositories(8)

Core Topic 3: Information Organization (141 subtopics):

Cluster 1. Metadata(753): Interoperability(metadata)(81), Metadata management (12), Metadata harvesting(24), Metadata aggregation(6) Metadata Extraction(19), Dublin Core(26), Standardization(67),Vocabulary control(24), Keywords(44), Controlled vocabulary(12), Terminologies(12), Thesaurus(44),Subject Headings(13), Indexing(348), Abstracting(110), Automatic Indexing(33), Science Citation Index(6), Video Indexing(7), Object Identifier(6),Url(7), RDF(14)

Cluster 2. Structured Documents(14): Markup languages(81), SGML(14), XML(330), HTML(119), Semi Structured Data(6),

Non-structured documents(2), Data Format(9)

Cluster 3. Bibliographic(161): Bibliographic data(6), Bibliographic records(11), Bibliographic control(2), Bibliographic information(10), Bibliographic database(26), Cataloging(30),Web Cataloguing(2), Union Catalogs(3),Bibliometric(10)

Cluster 4. Discovery(84): Resource Discovery(12), Information Discovery(11), Harvesting(44), Capturing(3), Information Gathering(5)

Navigation(74), Routing(14), Links(83), File Sharing(4), Data Exchange(10), Data Gathering(2), Data Dissemination(2), Data Exploration(6)

Data Sharing(18),Data Mining(253), Text Mining(32),Web Mining(9)

Cluster 5.Information Organization(General)(8): Classification(256), Classification systems(15), Taxonomy(47), Automatic

Classification(5), Document Classification(16), Categorization(46), Automatic categorization(4), Text categorization(26), Dewey Decimal

Classification(6), Topic maps(7)

Cluster 6. Conceptual(General)(47): Conceptual design(9), Conceptual graph(2), Conceptual frameworks(5),Conceptual discovery(1), Conceptual model(8),Concept map(14), Concept Space(6)

Cluster 7. Hierarchy(General)(24): Concept hierarchies(3), Topic hierarchy(2), Hierarchical systems(69),Hierarchical clustering(10),

Hierarchical Structure(14)

Cluster 8. Annotation(General)(125): Document annotation(4), Content annotation(2), Digital annotation(2),Image Annotation(10), Video Annotation(10)

Cluster 9. Compression(General)(87): Data compression(31), Image compression(53), Compression ratio(5),Compression algorithms(3) Cluster 10. Video processing(3): Video editing(4), Video recording(24), Video Streaming(15),Video Segmentation(8), Streaming Media(4) Rendering(16)

Cluster 11. Information analysis(263): Documents Analysis(15), Text Analysis(5), Data Analysis(31),Content analysis(22), Link Analysis(9), Visual Analysis(2), Speech Analysis(3), Citation Analysis(30)

Cluster 12. Recognition(General)(302): Character recognition(101), Handwriting recognition(7),Recognition process(4),OCR(25), Optical Music Recognition(4)

Cluster 13. Information processing(25): Text Processing(145), Image processing(223), Speech Processing(4), Administrative data processing(29), Government data processing(25),Natural language processing(124), Encoding(60), Information integration(21), Named Entities(12), Name disambiguation(19), Text Segmentation(5), Document Clustering(27), Text Clustering(6), Ranking(57), Authoring tool(9), Personalization(63), Personalized information(12), Summarization(31), Document Summarization(5), Interpretation(14), Data interpretations(2),Image Interpretation(3), Information Extraction(48), Text Extraction(6), Keyphrase extraction(8) 


\section{Core Topic 4: Information Retrieval (78 subtopics):}

Cluster 1. Information Retrieval(General)(1376): Bibliographic retrieval systems(113), Multimedia(IR)(121), Content Based Retrieval(135), Text retrieval(24), Document retrieval(26), Music retrieval(8), Image retrieval(181), Video retrieval(19), Interoperability(IR)(35),

Modeling(IR)(25), Personalisation(IR)(10), Retrieval effectiveness(7), Cross Lingual(IR)(19), Relevant documents(13), Retrieval techniques(4), Requirement Analysis(3), Document Frequency(5), String Matching(9)

Cluster 2. Multilingual(IR)(19): Multicultural(IR)(1), Asian Languages(IR)(4), Cross language(12), Chinese(IR)(5), Indian(IR(4),

Thailand(IR)(1), Language Model(5), Machine Translation(10)

Cluster 3. Search(General)(768): Search engines(496), Searching(386), Full text search(5), Search strategies(10), Search process(12), Meta search(11), Meta search engine(8), Web search(31), Web Search Engine(13), Information Seeking(58), Exploratory Search(8), Integrated search(2), Distributed search(5), Local search(4), Federated search(6), Video search(5), Music search(2), Image search(12), Search Method(8), Enterprise Search(4), Visual Search(3), Personalized Search(8), Interactive Search(3), Similarity Search(13)

Cluster 4 Query(General)(474): Query Language(298), Query Expansion(15), Query Processing(55), Query recommendations(1), Query suggestion(2), Query search(10), SQL query(3), Query Evaluation(1), Query Formulation(10), Query Refinement(5), Query Reformulation(3), Dynamic Query(4), Query Optimization(3)

Cluster 5. Browsing(General)(95): Video browsing(7), Document browsing(4), Web Browsing(3)

Cluster 6. Recommendation(General)(51): Recommendation system(17), Recommender systems(57)

Cluster 7. Filtering(General)(89):Filtering(Information Retrieval)(30), Information filtering(17), Collaborative filtering(42)

\section{Core Topic 5: Access (14 subtopics):}

Cluster 1. Access(General)(319): Access control(58), Open access(45), Multi-lingual access(3), Information Access(41) Accessibility(11), Data Access(22), Access methods(3), Wireless Access(2), Universal Access(5), Random Access(11), Multilingual Information Access(6), Internet Access(5), Connection(13)

\section{Core Topic 6: Human - Computer Interaction (61 subtopics):}

Cluster 1. Interactions(General)(279):Human-Computer Interaction(General)(168), Model(HCI)(20), User Interaction(10), Physical interactions(3), Bimanual interaction(2), Interaction design(13), Interactive computer graphics(34), Interactive Visualization(5), 3D interaction(5), Interactive Multimedia(5), Interactive System(1), Interaction Pattern(5), Interactive Display(1), Interaction Technique(4), Interactive Space(2)

Cluster 2. Human engineering(70): Technology Acceptance Model(11), Human Factors(36), Artificial intelligence(139), Human cognition(1) Machine Learning(49), Facial Expression(3), Face Recognition(17), Facial Features(3), Automatic Generation(2), Human Information Processing(9), Spatial Memory(2), Visually impaired(8), Automatic Speech Recognition(3)

Cluster 3. Visualization(General(262): Knowledge Representation(51), Information Representation(3), Multimedia Presentation(6), 2D(10), 3D(78), 3D Visualization(6), 3D model(6), Three Dimensional(120), Information Visualization(52), Visual Communication(29), Visual components(2), Geographical visualization(1), Contextual Information(9), Data Representation(7), Data Visualization(33), Graph Visualization(2), Visual Design(2), Visualization Technique(9)

Cluster 4. User Interfaces(790): User-Computer Interface(30), Object-oriented interfaces(1), User interface evaluation(3), Interface Design(35), Web Interface(25), Geographical visualization(1), Web Design(4), User-centric(6), User centred designs(3), Visual Interface(9), Sensor(57), Sensor Network(19)

\section{Core Topic 7: User Studies (59 subtopics):}

Cluster 1. Users(1208): User Communities(15), Students(267), Teachers(14), Children(30), Adults(14), Researcher(7), Scientists(10), Professor(2), Community Networks(3), Graduate students(10), Research groups(6), Web Community(4), Scholars(21), Scientific community(14), Blind Users(3)

Cluster 2. Usability(76): Usage(55), Usage patterns(6), Usability Evaluation(7), Usability engineering(30), Usability Testing(10), User Model(6), User Modeling(20), Weblogs(7), Adaptation(14), Log Data(7), Query Logs(8), Log Analysis(16), Transaction Log Analysis(5), Log Files(7),Localization(4)

Cluster 3. Information Needs(26): User needs(10), User interests(11), User requirements(12), User query(11), Needs Assessment(3) Cluster 4. User studies(General)(97): User evaluation(19), User profile(23), User behavior(19), Search behavior(10), Information Seeking Behavior(16), Information behavior(7), User communication(1), Feedback(78), User Feedback(4), Decision making(94), Decision support systems(41) Decision theory(26), Behavioral research(34), User Experience(18), User Preferences(6), User Testing(2), Human Memory(3), Cognitive Process(2), User Perception(7), User Satisfaction(7)

\section{Core Topic 8: Architecture - Infrastructure (144 subtopics):}

Cluster 1. Computing(General)(509): Client server(84), Distributed Computing(18),Distributed computer systems(236), Cluster Computer(2), Clustering(136),Parallel programming(33),Grid computing(153),Cloud computing(7),Ubiquitous computing(90), Scientific computing(5)

Cluster 2. Algorithms(General)(895): Learning Algorithm(53),Mathematical Model(457),Linear Algebra(34),Clustering Method(11),Search Algorithm(9),Schema Mapping(6),Probabilistic Model(11),Classification Algorithm(9),Computational methods(127),Computational tools(5) Cluster 3. Infrastructure(General)(95): Global Information Infrastructure(2), Cyberinfrastructure(10),Platform(70),Information Infrastructure(20)

Cluster 4. Software(General)(1203): Digital library software(28),Software infrastructure(5),Operating systems(129), Application software(6), Software engineering (367), Open Source Software(20), Open systems(50), Open Source(95), Open Source tools(2), Tools(256), Software Tool(17),Computer simulation(350), Simulation model(6), Controllers(29), Optimization(317), Artificial intelligence(139), Intelligent systems(20), Computer games(7),Software Design(38), Software Agent(26),Software Component(15),Software Platform(2), Software Requirements(2),Software Reuse(11)

Cluster 5. Architecture(General)(472): Digital Library Architecture(11),Digital library design(8),Computer architecture(208),Hardware architecture(4),Software architecture(36),Network architecture(20),Information architecture(11),Information Model(6), Open architecture(5), Computer engineering(9),Hardware(138),Peer to peer(50), Centralized architecture(2), Interoperability (Architecture)(184),Architectural Design(20),Vector spaces(30), Runtime environments(5),Time and Space (1), Groupware(14), Middleware(80),Service-oriented architecture(27), Design and Development(7) Cluster 6. Internet(699): Network(875),Web(1441), WWW(21), Web 2.0(33), Semantic Web(137), Web servers(30), Neural Network(69),Web 
Technology(28),Portals(127),Web Portal(11),Protocols(265)

Cluster 7. Data sets(80): Data Model(29), Data Warehousing(3), Data structures(305),Multiple data(5),Data Type(11),Database objects(6),Data Fusion(14),Data Center(4),Data Grid(24), Data Integrity(4)

Cluster 8. Digital Objects(83): Object oriented(213), Learning Object(41),Object oriented programming(196),Object-oriented modeling(2)

Cluster 9: Information systems(393),Database systems(1047),Information systems design(4),Database Design(5),System Design(28),

Digital library systems(88),Content management system(12),Multimedia systems(402),Design Principle(6), Replication(14),Entity

Resolution(5), Embedded systems(110),Data Management System(3),Database Development(1), Hybrid System(5),Spatial Data(22),Spatial

Distribution(2)

Cluster 10. Heterogeneous(General)(58): Heterogeneous Collections(4),Heterogeneous systems(8),Heterogeneous information(5),

Heterogeneous data(7), Large scale systems(64),Scalability(27),Extensibility(4),Large scale systems(52)

Cluster 11. Integration(General)(148): Digital library integration(12), Digital library federation(5), Integration systems(7)

Cluster 12. Distributed digital libraries(24): Distributed Collections(2),Distributed portal(3),Distributed systems(22), Distributed Data(9)

Distributed Database(84)

Cluster 13. Fuzzy systems(9): Fuzzy linguistic(9),Fuzzy logic(14),

Cluster 14. Agents(General)(165):Multi agent systems(50), Agent based(13),Intelligent agent(44)

Core Topic 9: Knowledge management (58 subtopics):

Cluster 1. Knowledge management(General)(185): Data management(38), Content management(45), Information management(411), Document Management(26),Knowledge organization systems(11), Content Management System(12), Multimedia data management(1),Knowledge based systems(150), Personal information management(10), Knowledge innovation(3), Scientific knowledge(8), Knowledge evolution(3), Knowledge map(4), Knowledge spaces(3), Knowledge basis(5), Domain knowledge(9), External knowledge(2), Information Space(14), Expert Knowledge(2),Knowledge Base(23), Expert System(28), Knowledge Work(1), Knowledge Network(8), Topic maps(6)

Cluster 2. Knowledge process(2): Knowledge building(1), Knowledge engineering(73), Knowledge gaps(1), Knowledge discovery(20), Knowledge acquisition(119), Knowledge organization(25), Knowledge representation(51),Knowledge Visualization(1), Knowledge sharing(22),Knowledge transfer(4), Knowledge retrieval(3), Knowledge Mining(2), Knowledge searching(1), Knowledge service(9), Knowledge distribution(1), Knowledge linking(1), Knowledge Translation(1),Knowledge map(4),Knowledge Extraction(6), Knowledge Exchange(1), Information Exchange(11), Information Sharing(22), Information Flow(4), Information communication(8), Knowledge communication(1)

Cluster 3. Collaboration(102): Collaborative work(5), Collaborative knowledge(4), Collaborative learning(11), Collaborative research(6), Collaborative network(2), Collaborative technologies(1)

\section{Core Topic 10: Digital Library Services (30 subtopics):}

Cluster 1. Services(General)(1134): Information dissemination(278), Information services(572), Library Services(84),Web services(179), Personal Digital Libraries(23), Personalized service(13), Personalized information services(3), Reference service(35), Service platforms(2), Online information services(8), Multimedia services(31), Data services(7), Database providers(1), Web search(31), Search services(14), Personal Digital Library(23), Telecommunication services(43), Mobile multimedia services(1), Service Provider(23), Service infrastructure(2), Catalog services(3), OPAC(6), Service Quality(11), Service System(12), Service Integration(6), Service Model(5), Reference Model(8), Reference Systems(4), Information Exchange(11)

Core Topic 11: Mobile technology (22 subtopics):

Cluster 1. Mobile library(3): Mobile services(5), Mobile content(1), Mobile information(3), Mobile users(6), Mobile access(4), Mobile reading(1), Mobile storytelling(1), Mobile learning(7)

Cluster 2. Mobile(General)(147): Mobility(15), Mobile devices(31), Mobile application(3), Mobile user interface(1), Mobile computing(22), Mobile communications(14), PDA(3),Wireless(63), Wireless networks(13), Wifi(2), 3G(2), Laptop(12)

Core Topic 12: Social Web(Web 2.0) (21 subtopics):

Cluster 1. Library 2.0(110): Librarian 2.0(15), Library user 2.0(1), Information literacy 2.0(2)

Cluster 2. Web 2.0(37) - Social Web(2): Social Networking(9),Social search(1), KnowledgeManagement 2.0(KM 2.0)(1), Social

networks(51), Social graph(1), Social Network Analysis(17), Social Media(5), Social Navigation(5)

Cluster 3. User generated content(3): Crowdsourcing(2), Wisdom of crowds(1), Social engagement(1), Folksonomy(7),Social tagging(12), Mashup(2)

Core Topic 13: Semantic Web (Web 3.0)(30 subtopics):

Cluster 1. Semantic digital library(21), Library 3.0(2)

Cluster 2. Semantic Web(137)-Web3.0(2): Semantic technology(16), Semantic search(4), Faceted search(7), Social semantics(2), Semantic Knowledge(1), Semantic interpretation(2), Semantic metadata(2), Semantic annotation(14), Semantic representation(1), Semantic resources(2), Semantic mapping(3), Semantic Zooming(4), Semantic Analysis(8), Semantic Model(4), Semantic Retrieval(5), Semantic Similarity(2), Semantic Web Service(10), Semantic Information(9), Semantic Relations(3)

Cluster 3. Ontologies(General)(258): Ontology services(1), Ontology development(2), Ontology semantics(21), Domain ontology(15), Formal Ontology(4), Ontology-based(19)

\section{Core Topic 14: Virtual Technologies (20 subtopics):}

Cluster 1. Virtual library(74), Library 3D(7), Virtual community(7), Virtual reference(16), 3D digital preservation(2), Virtual Learning(8) Cluster 2. Virtual(General)(541): Virtual reality(282), Virtual worlds(12), Virtual environments(33), Second Life(10), Virtualization(6), Virtual machines(50), Virtual laboratory(10), Virtual platform(1), Virtual Organization(8), Cybernetics(16), Web 3D(3), Virtual Instrument(10), 3D models(4)

Core Topic 15: Digital library management (53 subtopics):

Cluster 1. Policy(General)(96): Information Policy(6), Digital Library policy(1) 
Cluster 2. Planning(General)(145): Digital library planning(2), Strategic planning(45), Project Planning(9)

Cluster 3. Finance(10): Cost benefit analysis(12), Benefits(20), Pricing(5), Budget(14), Investment(23), Cost Effectiveness(41), Information Economics(1)

Cluster 4. Human resources(6): Staff(20), Digital librarians(5), Information professionals(14), Curators(2)

Cluster 5. Digital library management(21): Organization and management(23), Digital library performance(5), Digital library project(40), Project management(254), Systems development(14), Library constructions(1), Library organization(8),Work flows(19),

Systems development(13), Management Model(4), Management System(126), Management Strategy(2)

Cluster 6. Evaluation(General)(310): Performance Evaluation(16), Performance Measure(3), Digital library evaluation(30), Evaluation

Method(6), Evaluation framework(2), Heuristic Evaluation(2), Case studies(26), Field Study(8), Performance Metric(3),Performance

Improvement(3)

Cluster 7. Quality control(53): Quality Assurance(46), Quality Model(3), Quality Indicator(4), Quality Metric(3), Quality Assessment(7) Information Quality(7)

Cluster 8. Risk management(11): Risk assessment(18)

Core Topic 16: Digital Library Applications (64 subtopics):

Cluster 1. Research(General)(623): E-science(24), Scholarly communication(27), E-discovery(1), Research institutions(3),Cultural institutions(3), Information research(5), Design/methodology/approach(17), Citizen Science(3)

Cluster 2. Education(General)(645): Distance education(90), Teaching(197), Higher education(35), Academic libraries(110), National libraries(47), Public library(43), Educational digital libraries(33), Public Education(4), Instruction(95), Educational systems(3), School(50), Classroom(16), Online Education(3), Societies and institutions(298)

Cluster 3. Learning(General)(621): Learning systems(304), Learning Management System(6), Learning process(6), Learning objectives(3), Elearning(113), Learning Environment(28), Online Courses(6), Supervised Learning(6), Learning Activities(6),Learning Methods(6), Learning

Technology(7), Active Learning(7), Taxonomy learning(2)

Cluster 4. E-government(9): Offices(11), E-governance(4), Electronic Administration(1), Military(22), Health Care(68),

Hospital(23),Medicine(39), Children digital library(2), Disability digital library(1), Television(32), News(27), Film(11)

Cluster 5. Natural Science(23): Digital Earth(4),Geospatial(18), Information Industry(2), Environmental Monitoring(2), Astrophysics(4),

NASA(5), Life Sciences(9)

Cluster 6. Social Sciences(21): Art(52), Culture(31), Humanities(19), Museums(53)

Core Topic 17: Intellectual Property, Privacy, Security (28 subtopics):

Cluster 1. Intellectual Property(General)(55): Intellectual Property Protection(1), Copyright(107), Copyright Protection(12), Licensing(11), Authorship(9), Authoring(17), Rights Management(19), Digital Asset Management(DAM)(8), Digital Rights Management(DRM)(15), Copyright Law(16)

Cluster 2. Security(General)( 223): Security systems(17), Data security(10), Security model(1), Security policy(1), Security management(2),

Computer crime(27), Digital signatures(4), Network security(20), Validation(31), Authentication(22), Authorization(11), Cryptography(47),

Digital watermarking(33)

Cluster 3. Privacy(General)(38): Privacy policies(1), Privacy protection(6)

Core Topic 18: Cultural, Social, Legal , Economic Aspects (25 subtopics):

Cluster 1. Cultural(Aspects)(103): Cultural Heritages(70), Multicultural Digital Library(1), Heritage(96), Cross-cultural(8), Crosslanguages(15),Cross-cultural usability(4), Oral History(8)

Cluster 2. Social(Aspects)(221): Societies and institutions(285), Citizen science(3), Pedagogical(Aspects)(8), Knowledge economy(2),

Information society(13), Digital divide(9), Digital age(6), Globalization(3)

Cluster 3. Legal Aspects(17): Censorship(2), Trust(8), Copyright Law(16),Law(85)

Cluster 4. Economic (Aspects)(46): Electronic commerce(122),Business(42)

Core Topic 19: DL Research \& Development(48 subtopics):

Cluster 1. Interdisciplinary(General)(12): Interdisciplinary collaborations(1), Interdisciplinary research(4), Multidisciplinary(15), Computer Science(4752), Engineering(2618), Archive science(238), Social Sciences(2129), Mathematics(1342), Biochemistry-Genetics-Molecular Biology(648), Physics and Astronomy(252), Business, Management and Accounting(246),Information science(225),Decision Sciences(193), Academic (domains)(181), Medicine(121), Materials Science(120), Chemistry(104), Chemical Engineering(96), Earth and Planetary

Sciences(89), Industry (domains)(67), Government (domains)(58), Arts and Humanities(58), Energy(56), Museum(53), Health Professions(53), Agricultural and Biological Sciences(50), Environmental Science(42), Psychology(42), Nursing(24), Curation(23), Immunology and

Microbiology(22), Economics-Econometrics-Finance(20), Neuroscience(18), Pharmacology-Toxicology-Pharmaceutics(17), Dentistry(17),

Cluster 2. Research and Development(91): Digital library research(17), Digital library concepts(2), Digital library development(3),

Librarianship(11), Scholarship(4)

Cluster 3. International cooperation(20): International digital library(2), Global collaboration(3), International Collaboration(20), Universal

Digital Libraries(5), Digital library collaboration(1)

Core Topic 20: Information Literacy (20 subtopics):

Cluster 1. Information Literacy(General)(40): User education(4), Critical thinking(6), Critical evaluation(3), Lifelong learning(5), Media literacy(2), Computer literacy(1), Information overload(8), Decision making(90), Decision Process(2), Adult learning(2), Interactive learning environment(2), Learning communities(6), Information searching(7), Knowledge economy(2), Information society(13), Digital divide(9), Information ethics(3),Ethics(7), Reading(55)

Core topic 21: Digital Library education (5 subtopics):

Cluster 1. Digital Library education(General)(148):Digital Library Curriculum(1),Digital Library program(20), Digital Library training(2) Computer Science Education(9) 


\section{Discussions and Conclusion}

In comparison with the findings of Pomerantz et al (2006), Liew (2008) and the topic list of 15 core topics and 210 subtopics from JCDL (2001-2010), ECDL (1997-2010) and ICADL (1998-2010), the new DL Knowledge Map with 21 core topics and 1015 subtopics (1990-2010) provides the most up-to-date DL research topics and comprehensive view of DL research over two decades in a form of a categorised and structured set of concepts in the field of DL research. In the map, several new core topics, such as: Mobile Technology, Social Web(Web 2.0), Semantic Web (Web 3.0), and Virtual Technologies were added and grouped with many subtopics showing the increasing impacts of new technologies on DLs. While the core topics Architecture-Infrastructure (144 subtopics), Information Organization (141 subtopics), and Information Retrieval (78 subtopics) are the top 3 topics of research interest over 2 decades, Digital Library Education (5 subtopics) appears to be of less interest. Cross relationships between DL topics shows the interdisciplinary nature of DL research, e.g. subtopic Interoperability appears under core topics Architecture-Infrastructure and Information Retrieval; subtopic Services appears under core topics Mobile technology, and Semantic Web, etc.

The resulting DL knowledge map can be used for a variety of purposes, e.g. to show the major areas of research in DL, and to demonstrate the interdisciplinary nature of DLs. The DL knowledge map can also be used to develop a visual knowledge map of DL and it can be mapped onto a live database like SCOPUS, WebOfKnowledge, etc. , to facilitate information search and retrieval on DLs as well as more critical analysis of the field. It can also be used to develop a DL curriculum by identifying the traditional information science topics as well as new interdisciplinary knowledge and skills that are enriching DL as a field of study and thus should form part of a new DL curriculum.

\section{Further Work}

Only the methodology and findings of the DL knowledge map (1990-2010) have been reported in this paper. Further research based on the DL knowledge map is in progress showing the evolution of the DL as field of study. A visual knowledge map of DL is also being created using visual ontology development tool like Protégé to map the relationships among DL topics, authors, institutions, research domains, etc.

Acknowledgement: The ALA scholarship (AusAID funding) to support the PhD study of the author is gratefully acknowledged.

\section{REFERENCES}

1. ANSI/NISO Z39.19: 2005 Guidelines for the construction, format, and management of monolingual controlled vocabularies (2005)

2. Arms, W. Y. Digital Libraries. Cambridge, MA: The MIT Press (2000)

3. Borgman, C. L. From Gutenberg to the Global Information Infrastructure. Cambridge, MA: The MIT Press(2000)

4. Candela, L., Castelli, D., Pagano, P., Thanos, C., Ioannidis, Y., Koutrika, G., Ross, S., Schek, H. and Schuldt, H.. Setting the Foundations of Digital Libraries: The DELOS manifesto. D-Lib Magazine, 13(3/4).

http://www.dlib.org/dlib/march07/ castelli/03castelli.html (2007)

5. Chen, H. e. a. Survey and history of digital library development in Asia Pacific. Design and Usability of Digital

Libraries. London, Information Science Publishing: 1 - 22 (2005)

6. Chowdhury, G. G., Chowdhury, S. Introduction to Digital Libraries. London: Facet (2003)

7. Chowdhury, G. G. and S. Chowdhury. "Digital library research: major issues and trends." Journal of

Documentation 55(4): 409-448 (1999)

8. European Digital Library Conference. http://ecdlconference.isti.cnr.it/

9. International Conference on Asian Digital Libraries. http://www.icadl.org/

10. Joint Conference on Digital Libraries http://www.jcdl.org/index.shtml

11. Knowledge Management. http://en.wikipedia.org/wiki/Knowledge_management

12. Liew, C. L. "Digital library research 1997-2007." Journal of Documentation 65(2): pp. 245-266 (2008)

13. Lesk, M. Understanding Digital Libraries (Second ed.). San Francisco, CA: Morgan Kaufman Publishers (2004)

14. Pomerantz, J., B. M. Wildemuth, et al. "Curriculum Development for Digital Libraries." JCDL'06: 10. (2006)

15. SCOPUS. (2011). http://www.info.sciverse.com/scopus/about(2006)

16. Witten, I. H., \& Bainbridge, D. How to Build a Digital Library. San Francisco, CA: Morgan Kaufman Publishers (2003) 